\title{
Preneoplastic and neoplastic gallbladder lesions detected after cholecystectomy
}

\author{
Servet Kocaöz $z^{1}$, Gülay Turan ${ }^{2}$ \\ ${ }^{1}$ Department of General Surgery, Atatürk Training and Research Hospital, Bilkent, Ankara, Turkey \\ ${ }^{2}$ Department of Medical Pathology, Faculty of Medicine, Balıkesir University, Balıkesir, Turkey
}

Gastroenterology Rev 2019; 14 (3): 193-197

DOI: https://doi.org/10.5114/pg.2019.82675

Key words: cholecystectomy, laparoscopic, cholelithiasis, metaplasia.

\begin{abstract}
Address for correspondence: Servet Kocaöz MD, Department of General Surgery, Ankara Atatürk Eğitim ve Araştırma Hastanesi Üniversiteler mah., Bilkent cad., No: 1, 06800 Çankaya, Ankara, Turkey, phone: +90 (0312) 29125 25, fax: +90 (0312) 2912525 , e-mail: servet.kocaoz@gmail.com
\end{abstract}

\begin{abstract}
Introduction: Gallbladder cancer (GBC) is diagnosed often incidentally after cholecystectomies, with a rate of $0.1-3 \%$.

Aim: To review the clinical and morphological aspects of GBC and pre-neoplastic lesions in patients who underwent cholecystectomy.

Material and methods: A total of 5026 patients who underwent cholecystectomy between January 1, 2012 and December 31, 2017 were included in the study. Histological changes (acute cholecystitis, adenomyomatosis, xanthogranulomatous cholecystitis (XGC), polyps, antral metaplasia, intestinal metaplasia (IM), dysplasia, cancer, and others) in gallbladders (GB) from 5029 patients who underwent cholecystectomy for cholelithiasis were analysed.

Results: Gallbladder cancer was more common in women than in men $(14 / 4=3.5)$. A significant relation was found between cholelithiasis and GBC $(p=0.031)$. Of the patients with GBC, six had stage $1 \mathrm{a}(\mathrm{T} 1 \mathrm{a}+\mathrm{T} 1 \mathrm{~b})$, five had stage $1 \mathrm{~b}$ (T2NO), two had stage 2 (T3NO), three had stage $2 b$ (T1-3 N1), one had stage 3 (T4 NO), and one had stage 4 (T3N1M1). The IM was more common in females than in males $(K / E=3.3)$. A significant relationship was found between cholecystitis and IM $(p<0.001)$. A significant association was observed between IM and adenomyomatosis hyperplasia $(p=0.016)$.

Conclusions: In this study, it was observed that adenomyomatous hyperplasia and adenomatous polyp were associated with metaplastic changes in the GB pathologies, including XGC and follicular cholecystitis. It is thought that metaplasia-dysplasia may be associated with GBC. However, further studies on GB carcinogenesis are needed.
\end{abstract}

\section{Introduction}

Gallbladder cancer (GBC) is the most common cancer of the biliary system. The annual incidence is estimated to be 1 or 2 people per 100.000 [1, 2]. The GBC spreads very quickly and has a high mortality rate. The most important prognostic factor is the stage of the disease $[3,4]$. The GB stones are thought to cause a number of changes that cause atrophy, metaplasia, dysplasia, and ultimately cancer in the GB epithelium $[3,5]$. Because it does not cause any symptoms in the early period, the disease is diagnosed during surgery or at an advanced stage. The GBC was diagnosed in $0.1-3 \%$ of all cholecystectomies [2-6]. The survival rate is higher in early-stage GBC that has undergone cholecystectomy for cholelithiasis $[7,8]$. Because of the high risk of cancer, cholecystectomy is recommended in the presence of a GB polyps (GP) larger than $1 \mathrm{~cm}$ or when vascular pedicle is seen on ultrasonography (USG) [9]. Cellular differentiation begins in the GB due to inflammation and other factors added to the $G B$, resulting in intestinal metaplasia (IM). Increased cell damage causes dysplasia and ultimately carcinoma in situ [10, 11]. Development of dysplasia-carcinoma in the GB is a long process. Preliminary lesions, environmental factors, and risk factors need to be combined $[12,13]$.
Aim
In our study, we aimed to review the clinical and morphological aspects of pre-neoplastic lesions of the GB in patients undergoing cholecystectomy and to anal- yse the results and our results in cancers observed in this organ. 


\section{Material and methods}

A total of 5026 patients who underwent cholecystectomy between January 1, 2012 and December 31, 2017 were included in the study. Medical records and histopathological findings of these patients were evaluated retrospectively. All samples taken after surgery included in these reports were fixed in $10 \%$ phosphate-buffered formaldehyde and stored in paraffin. In addition, the sections obtained from the samples were stained with haematoxylin-eosin and PAS/Alcian Blue $(\mathrm{pH}=2.5)$ and then prepared for routine light microscopy. Then, all the samples were evaluated by an expert pathologist. In each case, the presence of stones in the lumen of the GB, type of inflammation in the wall of the sac, cholesterolosis, polyps, metaplasia, dysplasia, cancer, and other lesions were recorded. It was compared with the previous histopathology report and re-evaluated in the presence of incompatibility. Demographic information (age, sex) of the patients was reviewed.

\section{Ethical considerations}

Before initiating the present study, the researchers gained the approval of Balıkesir University Medical Faculty Clinical Research Ethics Committee (Decision no: 196, date: 05.12.2018) and the written permission of Balıkesir Atatürk City Hospital where the research was conducted.

Table I. Histopathological changes of the gallbladder

\begin{tabular}{lcc} 
Parameter & $\begin{array}{c}\text { Number of } \\
\text { patients }\end{array}$ & Percentage \\
\hline Chronic cholecystitis & 2411 & 47.97 \\
\hline Acute cholecystitis & 757 & 15.06 \\
\hline Gangrenous cholecystitis & 67 & 1.33 \\
\hline Cholesterolosis & 1209 & 24.05 \\
\hline Xanthogranulomatous cholecystitis & 122 & 2.43 \\
\hline Follicular cholecystitis & 36 & 0.72 \\
\hline Eosinophilic cholecystitis & 17 & 0.34 \\
\hline Acalculous cholecystitis & 41 & 0.82 \\
\hline Adenomyomatous hyperplasia & 153 & 3.04 \\
\hline Cholesterol polyp & 102 & 2.03 \\
\hline Hyperplasic polyp & 8 & 0.16 \\
\hline Tubular adenoma & 25 & 0.5 \\
\hline Inflammatory polyp & 11 & 0.22 \\
\hline Antral type metaplasia & 628 & 12.5 \\
\hline Intestinal metaplasia & 128 & 2.55 \\
\hline Dysplasia & 49 & 0.97 \\
\hline Cancer & 18 & 0.35 \\
\hline
\end{tabular}

\section{Statistical analysis}

The data obtained from this study were transferred to the SPSS program (Version 25.0; SPSS Inc., Chicago, IL, USA) and then analysed. The number, percentage, mean, and standard deviation values were utilised for the evaluation of descriptive statistics. The chi-square test $\left(\chi^{2}\right)$ was used to define the correlations among the clinical and pathological datum.

\section{Results}

Of the 5026 patients (1228) who underwent open and laparoscopic cholecystectomy (LC), 24.43\% were male (3798) and $75.56 \%$ were women. The mean age of male patients was $54.11 \pm 13.77$ years, and the mean age of female patients was $51.27 \pm 14.43$ years. It was found that female patients had GB disease at an earlier age than male patients $(p=0.002)$. Histopathological examination of the patients found acute stone cholecystitis in 15.06\% (757), acute gangrenous cholecystitis in $1.33 \%(67)$, acalculous acute cholecystitis in $0.82 \%$ (41), XGC in $2.43 \%$ (122), follicular cholecystitis in $0.72 \%$ (36), eosinophilic cholecystitis in $0.34 \%$ (17), GB polyps in $5.95 \%$ (299), cholesterolosis in $24.05 \%$ (1209), and chronic stone cholecystitis in $47.97 \%$ (2411). In addition, operated patients were found to have antral type metaplasia in $12.5 \%$ (628), intestinal metaplasia in $2.55 \%$ (128), dysplasia in $0.97 \%$ (49), and adenocarcinoma in $0.35 \%$ (18). Of the 299 patients detected with polyps in the GB of histopathology, $0.22 \%$ (11) had inflammatory polyps, 3.04\% (153) had adenomyomatous hyperplasia, $0.16 \%$ (8) had hyperplastic polyps, $0.5 \%$ (25) had tubular adenomas, and 2.03\% (102) had cholesterol polyps (Table I). In patients with GBC, the mean age of men was $63.25 \pm 11.35$ years and the mean age of women was $66.21 \pm 12.15$ years. GBC was higher in women than in men $(14 / 4=3.5)$. A significant correlation was found between cholelithiasis and GBC $(p=0.031)$. Six of the patients with $G B C$ had stage I $A$ $(\mathrm{T} 1 \mathrm{a}+\mathrm{T} 1 \mathrm{~b})$, five had stage $1 \mathrm{~b}(\mathrm{~T} 2 \mathrm{~N} 0)$, two had stage II A (T3N0), three had stage $2 \mathrm{~b}(\mathrm{~T} 1-3 \mathrm{~N} 1)$, one had stage III

Table II. Stages of patients with gallbladder cancer

\begin{tabular}{lcc} 
Stage & Number of patients & Percentage \\
\hline I A & 6 & 33.33 \\
\hline I B & 5 & 27.78 \\
\hline II A & 2 & 11.11 \\
\hline II B & 3 & 16.67 \\
\hline III & 1 & 5.56 \\
\hline IV & 1 & 5.56
\end{tabular}


(T4 N0), and one had stage IV (T3N1M1) (Table II). In addition, IM was higher in women than in men $(K / E=3.3)$. There was no relationship between $I M$ and gender $(p=0.595)$. Similarly, no correlation was found between age and IM ( $p=0.451)$. A meaningful relationship was found between cholecystitis and IM $(p<0.001)$. A meaningful association was observed between IM and adenomyomatous hyperplasia $(p=0.016)$. A meaningful relationship was found between the presence of cholesterol and IM comorbidity $(p<0.001)$.

\section{Discussion}

Cholecystectomy is a common method for the treatment of GB diseases. Laparoscopic cholecystectomy (LC) is preferred for reasons such as shorter operation time, lower complication and mortality rates, and early return of patients to daily life activities during the postoperative period. However, open cholecystectomy is performed in the presence of factors such as cancer suspicion in the GB, congenital anomalies of the biliary tract and gallbladder, liver cirrhosis, bleeding disorders, especially third trimester of pregnancy, and adhesions between GB and surrounding tissue. In order to detect clinically asymptomatic malignancies, it is recommended that a sufficient number of samples be taken from cholecystectomy material [14].

The most common malignant tumour (88\%) in the GB is adenocarcinoma. Squamous cell carcinoma, adenosquamous carcinoma, small cell carcinoma, undifferentiated carcinoma, and other tumours can be seen less frequently [15]. Clinically, a significant proportion of GB malignancies are asymptomatic and incidentally found in the histopathological examination (47\%) [15]. In our study, GBC prevalence was found to be $0.35 \%$ in the histopathology of LC patients. In the study of Pitt et al. the prevalence of GBC was found to be $0.19 \%$. In a similar study by Martins-Filho et al. the prevalence of GBC was found to be $0.34 \%$. Our study was consistent with the literature $[16,17]$. Utsumi et al. found the prevalence of GBC to be $2.3 \%$. It has been reported that factors such as Asian or African American race, age over 65 years, female gender, obesity, biliary stone, polypoid lesions, high alkaline phosphatase level, and nutrition can cause a high prevalence [18]. In our study, among GBC patients, $78 \%$ (14) were female, 67\% (12) had cholelithiasis, and $11 \%$ (2) had GB polyps. Cholelithiasis causes inflammation of the $G B$ as a result of chronic irritation, followed by the development of mucosal dysplasia and eventually development of carcinoma. In the early stages, GB wall thickening is seen in both cholecystitis and cancer. It is therefore difficult to distinguish. In the case of suspected $G B C$, intraoperative frozen section examination should be performed [19]. In our study, two cases had an intraop- erative frozen pathologic examination. Subsequently, GB bed resection and hepatic peduncle lymphadenectomy were performed. The only curative treatment for GBC is surgical resection. LC is sufficient in stage pT1a. Six patients in our study had LC. GB bed resection and hepatic peduncle lymphadenectomy should be performed in stage pT1b. Five patients with stage pT1b underwent resection of the GB bed and lymphadenectomy. Resection of hepatic segment $4 a$ and 5 , hepatic peduncle lymphadenectomy, and additional radical surgical procedures should be performed in stage pT2 $[18,20]$.

The relationship among cholelithiasis and GBC is well known. A relationship between cholecystolithiasis and XGC, adenomyomatous hyperplasia and antral metaplasia has been reported [21, 22]. In our study, a significant correlation was found between cholelithiasis and GBC ( $p=0.031)$. The high risk of GBC in women is partly explicable by the proportion of higher cholesterol cytolysis in men than in women. Longer exposure to female sex hormones (such as oestrogen and progesterone) may increase the risk of GBC [23].

Acute cholecystitis and gangrenous cholecystitis were more common in men. The results are similar in other studies [5]. In a male patient with acute cholecystitis, GBC was determined. Non-infectious GB adenomyomatous hyperplasia is defined as a precancerous lesion. Cases of GBC associated with adenomyomatous hyperplasia have been reported [24].

$X G C$ is a rare purulent and devastating GB disease that can propagate to neighbouring structures and can be mingled with cancer. Microscopically, purulent cell infiltration is seen in the GB wall, as well as the presence of a large number of foamy macrophage groups. XGC is detected in approximately $2 \%$ of cholecystectomies. Men and women are seen as equal and often with gallstones [25]. In our study, this rate was found at $2.43 \%$. The GBC was reported as determined in $9 \%$ to $12 \%$ of patients with XGC $[25,26]$. In our study, cancer was detected in a patient with XGC. Cholecystitis in which lymphoid follicles with a germinal centre filling the lamina propria are seen is known as follicular cholecystitis. In this study, a patient with follicular cholecystitis was also diagnosed with cancer.

Metaplastic changes are associated with cancer. There are two metaplastic changes in the GB: antral and intestinal. Metaplastic changes and dysplasia increase with age and are most common in patients over 50 years of age [11]. In our study, metaplastic changes were found in $78.7 \%$ (96) of patients with XGC, in 75\% (27) of patients with follicular cholecystitis, and in 33.99\% (52) of patients with adenomyomatous hyperplasia.

In our study, 18 patients had adenomatous polyps. Adenomatous polyps show cancer transformation. How- 
ever, malignant transformation of adenoma is rare. The evolution of cancer is connected with the size of the adenomatous polyps [5]. In our study, metaplastic changes were found in $68 \%$ (17) of the patients with adenoma.

Cholesterolosis occurs as a result of the collection of cholesterol esters and triglycerides in the cytoplasm of macrophages in the GB wall. Macroscopically, it is seen as yellow lines (strawberry sac) on the mucosal surface or as polypoid lesions of $0.1-0.5 \mathrm{~cm}$. It is thought that the serum cholesterol level is due to its height. However, it has been shown not to have any association with high cholesterol levels [27]. Polypoid shaped ones are also known as cholesterol polyps and are the most common polyp type in the GB. It is possible to observe at the same time various purulent, reactive, and neoplastic polyps in the GB mucosa. In the presence of polyps larger than $10 \mathrm{~mm}$ in the $\mathrm{GB}$, the presence of GBC should be investigated [28]. In our study, cholesterol polyp was detected in $2.03 \%$ of patients who underwent LC. A meaningful association was found between the presence of cholesterosis and metaplastic changes $(p<0.001)$.

On ultrasonography, when the GB wall is seen to be thickened (greater than $3 \mathrm{~mm}$ ), approximately $50 \%$ of these patients appear to have XGC. Because of the high risk of cancer in these patients, $L C$ is recommended. Again, in patients with large gallstones in the GB (greater than $3 \mathrm{~cm}$ ), prophylactic cholecystectomy in the presence of porcelain gallbladder and stemless polyps (greater than $1 \mathrm{~cm}$ ) is reported to reduce the $\mathrm{GBC}$-related mortality rate [27]. However, Lee et al. reported no significant correlation between LC rate, GBC incidence, and mortality [28]. There is insufficient evidence to suggest that prophylactic LC should be performed in patients with asymptomatic cholecystitis to diminish the incidence and mortality of rare GBC $[29,30]$.

\section{Conclusions}

In this study, chronic cholecystitis, XGC, follicular cholecystitis, adenomyomatous hyperplasia, adenomatous polyp, and even cholesterolosis were associated with metaplastic changes. It is thought that metaplasia-dysplasia may be associated with GBC. However, further studies on GB carcinogenesis are needed.

\section{Conflict of interest}

The authors declare no conflict of interest.

\section{References}

1. Shih SP, Schulick RD, Cameron JL, et al. Gallbladder cancer: the role of laparoscopy and radical resection. Ann Surg 2007; 245: 893-901.
2. Hundal R, Shaffer EA. Gallbladder cancer: epidemiology and outcome. Clin Epidemiol 2014; 6: 99-109.

3. Lazcano-Ponce EC, Miquel JF, Muñoz N, et al. Epidemiology and molecular pathology of gallbladder cancer. CA Cancer J Clin 2001; 51: 349-64.

4. Zhang WJ, Xu GF, Zou XP, et al. Incidental gallbladder carcinoma diagnosed during or after laparoscopic cholecystectomy. World J Surg 2009; 33: 2651-6.

5. Roa I, de Aretxabala X, Araya JC, Roa J. Preneoplastic lesions in gallbladder cancer. J Surg Oncol 2006; 93: 615-23.

6. Meirelles-Costa AL, Bresciani CJ, Perez RO, et al. Are histological alterations observed in the gallbladder precancerous lesions? Clinics (Sao Paulo) 2010; 65: 143-50.

7. Cavallaro A, Piccolo G, Panebianco V, et al. Incidental gallbladder cancer during laparoscopic cholecystectomy: managing an unexpected finding. World J Gastroenterol 2012; 18: 4019-27.

8. Sun CD, Zhang BY, Wu LQ, Lee WJ. Laparoscopic cholecystectomy for treatment of unexpected early-stage gallbladder cancer. J Surg Oncol 2005; 91: 253-7.

9. Aloia TA, Járufe N, Javle $M$, et al. Gallbladder cancer: expert consensus statement. HPB (Oxford) 2015; 17: 681-90.

10. Mukhopadhyay S, Landas SK. Putative precursors of gallbladder dysplasia: a review of 400 routinely resected specimens. Arch Pathol Lab Med 2005; 129: 386-90.

11. Fernandes JE, Franco MI, Suzuki RK, et al. Intestinal metaplasia in gallbladders: prevalence study. Sao Paulo Med J 2008; 126: 220-2.

12. Lewis JT, Talwalkar JA, Rosen CB, et al. Prevalence and risk factors for gallbladder neoplasia in patients with primary sclerosing cholangitis: evidence for a metaplasia-dysplasia-carcinoma sequence. Am J Surg Pathol 2007; 31: 907-13.

13. Khan MR, Raza SA, Ahmad Z, et al. Gallbladder intestinal metaplasia in Pakistani patients with gallstones. Int J Surg 2011; 9: 482-5.

14. Amiraslanov A, Zade KY, Musayev J. Histopathological profile of the gallbladder in cases who have undergone laparoscopic cholecystectomy. Marmara Med J 2015; 28: 32-37.

15. Duffy A, Capanu M, Abou-Alfa GK, et al. Gallbladder cancer (GBC): 10-year experience at Memorial Sloan-Kettering Cancer Centre (MSKCC). J Surg Oncol 2008; 98: 485-9.

16. Pitt SC, Jin LX, Hall BL, et al. Incidental gallbladder cancer at cholecystectomy: when should the surgeon be suspicious? Ann Surg 2014; 260: 128-33.

17. Martins-Filho ED, Batista TP, Kreimer F, et al. Prevalence of incidental gallbladder cancer in a tertiary-care hospital from Pernambuco, Brazil. Arq Gastroenterol 2015; 52: 247-9.

18. Utsumi M, Aoki H, Kunitomo T, et al. Evaluation of surgical treatment for incidental gallbladder carcinoma diagnosed during or after laparoscopic cholecystectomy: single center results. BMC Res Notes 2017; 10: 56.

19. Kwon AH, Imamura A, Kitade H, Kamiyama Y. Unsuspected gallbladder cancer diagnosed during or after laparoscopic cholecystectomy. J Surg Oncol 2008; 97: 241-5.

20. Watson H, Dasari B, Wyatt J, et al. Does a second resection provide a survival benefit in patients diagnosed with incidental T1b/T2 gallbladder cancer following cholecystectomy? HPB (Oxford) 2017; 19: 104-7. 
21. Martínez-Guzmán G, de la Rosa-Bayón J. Neoplasms and dysplasias of the gallbladder and their relationship with lithiasis. A case-control clinicopathological study. Rev Gastroenterol Mex 1998; 63: 82-8.

22. Khanna R, Chansuria R, Kumar M, Shukla HS. Histological changes in gallbladder due to histological changes in gallbladder due to stone disease. Indian J Surg 2006; 68: 201-4.

23. Shukla VK, Chauhan VS, Mishra RN, Basu S. Lifestyle, reproductive factors and risk of gallbladder cancer. Singapore Med J 2008; 49: 912-5.

24. Cariati A, Piromalli E, Cetta F. Gallbladder cancers: associated conditions, histological types, prognosis, and prevention. Eur J Gastroenterol Hepatol 2014; 26: 562-9.

25. Spinelli A, Schumacher G, Pascher A, et al. Extended surgical resection for xanthogranulomatous cholecystitis mimicking advanced gallbladder carcinoma: a case report and review of literature. World J Gastroenterol 2006; 12: 2293-6.

26. Kwon AH, Sakaida N. Simultaneous presence of xanthogranulomatous cholecystitis and gallbladder cancer. J Gastroenterol 2007; 42: 703-4

27. Dairi S, Demeusy A, Sill AM, et al. Implications of gallbladder cholesterolosis and cholesterol polyps? J Surg Res 2016; 200: 467-72.

28. Lee H, Kim K, Park I, et al. Preoperative predictive factors for gallbladder cholesterol polyp diagnosed after laparoscopic cholecystectomy for polypoid lesions of gallbladder. Ann Hepatobiliary Pancreat Surg 2016; 20: 180-6.

29. Kapoor VK. Cholecystectomy in patients with asymptomatic gallstones to prevent gall bladder cancer: the case against. Indian J Gastroenterol 2006; 25: 152-4.

30. Le MD, Henson D, Young $H$, Albores-Saavedra J. Is gallbladder cancer decreasing in view of increasing laparoscopic cholecystectomy? Ann Hepatol 2011; 10: 306-14.

Received: 9.01.2019

Accepted: 18.01.2019 